\title{
Preparation of Elastomeric Ionene Polymers Containing 4,4'-Bipyridinium or 1,2-Bis(4-pyridinium)ethylene Ring and the Conductivity of Their TCNQ Salts
}

\author{
Masayoshi WATANABE, Nobuhiro TONEAKI, and Isao SHINOHARA \\ Department of Polymer Chemistry, Waseda University, \\ Ohkubo, Shinjuku-ku, Tokyo 160, Japan.
}

(Received August 14, 1981)

\begin{abstract}
Elastomeric ionene polymers containing 4,4'-bipyridinium (BP) or 1,2-bis(4pyridinium)ethylene (BPE) ring were prepared by the Menschutkin reaction of $4,4^{\prime}$-bipyridine or 1,2-bis(4-pyridyl)ethylene with an alkyliodide-terminated prepolymer derived from poly(propylene glycol) (PPG). Resistivity $(\rho)$ and activation energy for conduction $\left(E_{\mathrm{a}}\right)$ were measured for the 7,7,8,8-tetracyanoquinodimethane (TCNQ) salts of these elastomeric ionenes. The values of $\rho$ were $26.7 \Omega \mathrm{cm}$ and $24.8 \Omega \mathrm{cm}$, and the values of $E_{\mathrm{a}}$ were $0.077 \mathrm{eV}$ and $0.079 \mathrm{eV}$ for (BP-PPG)-TCNQ complex salt and (BPE-PPG)-TCNQ complex salt, respectively. The complex salts were processible from solutions in the form of flexible films. A microheterogeneous structure of continuous conduction columns composed of $\mathrm{TCNQ}^{-}$and $\mathrm{TCNQ}^{\circ}$ and the poly(oxypropylene) chain brought about a high conductivity and favourable mechanical properties. These TCNQ salts were stable in an ambient condition, and the values of $\rho$ at the storage time of about 300 days were nearly twice as large as the initial values.

KEY WORDS 7,7,8,8-Tetracyanoquinodimethane (TCNQ) / Elastomeric Ionene / 4,4'-Bipyridinium / 1,2-Bis(4-pyridinium)ethylene / Poly(propylene glycol) / Conductivity / Complex Salt / Flexible Film /
\end{abstract}

The complex composed of 7,7,8,8-tetracyanoquinodimethane (TCNQ) and polycation is known as an organic semiconducting polymer, soluble in organic solvents and processible in film form. ${ }^{1}$ Electronic conductivity of polycation-TCNQ salts is affected by polycation structure. Complex salts, obtained by doping $\mathrm{TCNQ}^{\circ}$ into simple salts, show higher conductivity than simple salts. The authors have shown that ionene polymers containing $\mathrm{N}^{+}$ atoms in the main chain, especially, whose intervals between $\mathrm{N}^{+}$atoms are fixed to $6-8 \AA$ by rigid ring structure, allowed the complex formation of $\left[\mathrm{TCNQ}^{\circ}\right] /\left[\mathrm{TCNQ}^{-}\right]=1.0 .^{2,3}$ This brings about simultaneously a lowering of the Coulomb repulsion between carrier electrons and an increase in the overlap of $\pi$-electrons of TCNQ molecules. ${ }^{3}$ Consequently, the complex salt of these polycations shows high conductivity. It was also found that the polycations containing highly polarizable aromatic heterocyclic amines reduced the Coulomb repulsion between carrier electrons and contributed to the high conductivity of their complex salts. ${ }^{4}$ The ionene polymers containing 4,4'-bipyridinium (BP) or 1,2-bis(4-pyridinium)ethylene (BPE) rings satisfy the above-mentioned conditions, and the conductivity of these TCNQ salts was highest among the polycation-TCNQ complex salts obtained. ${ }^{5,6}$ However, the films obtained are generally brittle and lack mechanical strength as polymeric materials. In order to be suitable for industrial application, an electrically conductive polymer should be flexible and capable of being shaped by a normal precess.

In this study, elastomeric ionene polymers containing BP or BPE rings were prepared in order to obtain high conductive and good processible polycation-TCNQ salts. The complex salts of these elastomeric ionenes were found to show resistivity $(\rho)$ of about $25 \Omega \mathrm{cm}$ and activation energy for conduction of $0.07-0.08 \mathrm{eV}$, and to be processable into flexible films from the solution. The change in $\rho$ with time was also investigated. 


\section{EXPERIMENTAL}

\section{Purification of Reagents and Solvents}

4,4'-Bipyridine and 1,2-bis(4-pyridyl)ethylene were recrystallized from methanol. Poly(propylene glycol) was obtained from Wako Pure Chemical Industry Co., Ltd. and used as obtained. The molecular weight determined from viscosity measurement was found to be 1290. 2,4-Toluylenediisocyanate (TDI) and 2-dimethylaminoethanol (DMAE) were purified by distillation under reduced pressure of $87^{\circ} \mathrm{C} / 4 \mathrm{mmHg}$ and $53^{\circ} \mathrm{C} / 56 \mathrm{mmHg}$, respectively. 1,5-Diiodopentane was obtained from Eastman Kodak Co., Ltd. and used without further purification. $\mathrm{TCNQ}^{\circ}$ was recrystallized twice from purified acetonitrile. LiTCNQ was synthesized according to the previously established procedure. ${ }^{7}$

Methanol was purified by distillation over calcium oxide. $N, N$-Dimethylformamide was treated with $4 \AA$ molecular sieves and then distilled $\left(60^{\circ} \mathrm{C} /\right.$ $30 \mathrm{mmHg}$ ). Acetonitrile was refluxed over phosphorus pentoxide for $2 \mathrm{~h}$ and distilled twice.
Preparation of Elastomeric Ionene Polymers

The elastomeric ionene polymers (BP-PPG, BPEPPG) were prepared according to the procedure shown in Figure 1.

PPG was allowed to react with TDI at $60^{\circ} \mathrm{C}$ for $18 \mathrm{~h}$ in a bulk system with stirring, at a molar ratio of $[\mathrm{TDI}] /[\mathrm{PPG}]=2.0$. Proceeding of the reaction was observed by infrared spectra, and completion of the reaction was confirmed by titration of the isocyanate groups. The product (I) was cooled to room temperature and a stoichiometric amount of DMAE was added. The contents were heated to $60^{\circ} \mathrm{C}$ again, and the reaction was allowed to continue for $2.5 \mathrm{~h}$ with stirring. The completion of the reaction was confirmed by the disappearance of the characteristic absorption of isocyanate groups in the infrared spectra. Unreacted DMAE was completely removed by a vacuum evaporator to yield a dimethylamino-terminated prepolymer (II). Molecular weight of the prepolymer (II) was determined by titration of the amino groups. Afterwards, the prepolymer (II) $\left(0.1 \mathrm{moll}^{-1}\right)$ was allowed to

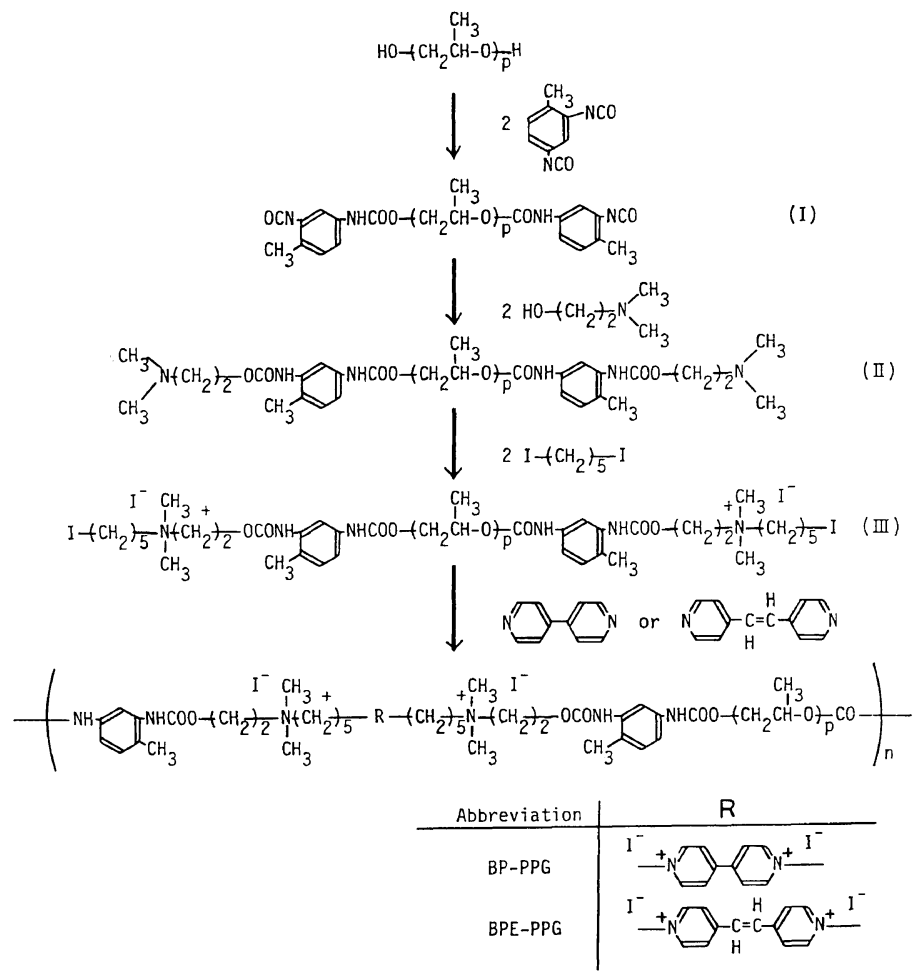

Figure 1. Procedure for preparation of elastomeric ionene polymers. 
react with 1,5 -diiodopentane $\left(0.2 \mathrm{moll}^{-1}\right)$ in methanol at $60^{\circ} \mathrm{C}$ for $24 \mathrm{~h}$, in a flask equipped with a condenser and a stirrer. Successively, the stoichiometric amount of 4,4'-bipyridine or 1,2-bis(4pyridyl)ethylene was added to the methanol solution of the prepolymer (III) as a chain extender, and the polymerization proceeded by the Menschutkin reaction for $150 \mathrm{~h}$. The reaction solution was diluted by methanol and poured into a large excess of diethyl ether. Precipitates were collected and dried in vacuo at $80^{\circ} \mathrm{C}$ for $48 \mathrm{~h}$. Yield: BPPPG, $76.3 \%$; BPE-PPG, $77.9 \%$.

\section{Preparation of TCNQ Salts}

Simple salts were obtained by a counter ion exchange reaction between the elastomeric ionenes and LiTCNQ. A methanol solution of LiTCNQ was dropped into a methanol solution of the elastomeric ionene. The mixed solution (elastomeric ionene: $1.1 \times 10^{-2}$ unit $\mathrm{moll}^{-1}$, LiTCNQ: $4.8 \times 10^{-2}$ moll $1^{-1}$ ) was stirred for $30 \mathrm{~min}$ at room temperature under a nitrogen atmosphere. The simple salts precipitated from the solution were filtered off, thoroughly washed with methanol and diethylether, and dried in vacuo. It was apparent from electronic spectra that the iodine ion of the elastomeric ionene was completely exchanged by $\mathrm{TCNQ}^{-}$of LiTCNQ. ${ }^{8}$ Yield: (BP-PPG)-TCNQ simple salt, $69.9 \%$; (BPE-PPG)-TCNQ simple salt, $66.3 \%$.

Complex salts were obtained by evaporating DMF under reduced pressure after dissolving the simple salt and $\mathrm{TCNQ}^{\circ}$ in DMF. They were dried in vacuo at $70^{\circ} \mathrm{C}$ for $72 \mathrm{~h}$.

\section{Electrical Measurement}

A DMF solution of TCNQ salts was concentrated to about $0.2 \mathrm{~g} \mathrm{ml}^{-1}$ by evaporating DMF and casted on clean glass plates. The solvent was completely removed under reduced pressure at $50^{\circ} \mathrm{C}$ for $24 \mathrm{~h}$ at first and at $70^{\circ} \mathrm{C}$ for $48 \mathrm{~h}$ at the last. Electrodes were made with the silver paste on the sample films of the glass substrates. Resistance was measured by the four probe method with direct current, using a Keithley 616 digital electrometer.

Activation energy for conduction $\left(E_{\mathrm{a}}\right)$ was calculated from the temperature dependence of the resistance, ranging from $25^{\circ} \mathrm{C}$ to $100^{\circ} \mathrm{C}$, using the equation,

$$
R=R_{0} \exp \left(E_{\mathrm{a}} / k T\right),
$$

where $k$ is the Boltzmann constant $\left(\mathrm{eV} \mathrm{K}^{-1}\right), T$ is the absolute temperature $(\mathrm{K})$, and $R_{0}$ is a constant $(\Omega)$.

\section{$X$-ray Diffraction Patterns}

$X$-ray diffraction patterns were measured with a Rigaku-Denki D-3F X-ray diffractometer using a $\mathrm{Cu}$ target.

\section{Spectral Measurements}

Infrared spectra were obtained with a JASCO IRA-1 diffraction grating infrared spectrophotometer, using $\mathrm{NaCl}$ plates. Electronic spectra was obtained with a Union Giken SM-401 spectrometer in acetonitrile.

\section{RESULTS AND DISCUSSION}

\section{Preparation of Elastomeric Ionene Polymers}

Figure 2 shows the infrared spectra of PPG, prepolymer (I) and prepolymer (II). As PPG reacted with TDI, the absorption at $3470 \mathrm{~cm}^{-1}$, being assignable to terminal hydroxy groups of PPG, gradually faded out. Instead, absorption appeared at $3300 \mathrm{~cm}^{-1}$ and $1720 \mathrm{~cm}^{-1}$ as shown in Figure 2(a), (b) and these bands are assinable ${ }^{9}$ to hydrogen bonded $\mathrm{N}-\mathrm{H}$ groups and carbonyl groups of urethane bonds respectively. After $18 \mathrm{~h}$ following the onset of the reaction, the absorption at $3470 \mathrm{~cm}^{-1}$ completely disappeared. The functionality of the isocyanate end-capped prepolymer (I) was found to be 1.99. When DMAE was added to the prepolymer (I), the absorption at $2280 \mathrm{~cm}^{-1}$, due to the iso-

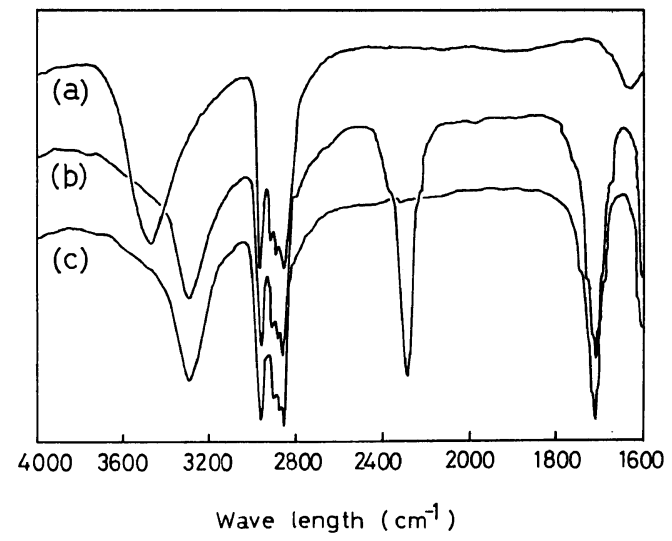

Figure 2. Infrared spectra of PPG, prepolymer (I) and prepolymer (II): (a), PPG; (b), prepolymer (I); (c), prepolymer (II). 
Table I. Elemental analyses and intrinsic viscosities of elastomeric ionenes

\begin{tabular}{|c|c|c|c|c|c|}
\hline \multirow{2}{*}{ Sample } & \multicolumn{4}{|c|}{ Elemental analyses $/ \%$} & \multirow{2}{*}{$\frac{[\eta]^{\mathrm{b}}}{\mathrm{dl} \mathrm{g}^{-1}}$} \\
\hline & $\mathrm{C}$ & $\mathrm{H}$ & $\mathbf{N}$ & $\mathrm{I}^{-\mathrm{a}}$ & \\
\hline BP-PPG & $\begin{array}{lc}\text { Found } 50.6 \\
\text { Calcd }(51.7)\end{array}$ & $\begin{array}{c}7.27 \\
(7.51)\end{array}$ & $\begin{array}{c}4.50 \\
(4.28)\end{array}$ & $\begin{array}{c}19.7 \\
(19.3)\end{array}$ & 0.270 \\
\hline BPE-PPG & $\begin{array}{l}\text { Found } 50.4 \\
\text { Calcd }(51.3)\end{array}$ & $\begin{array}{c}7.42 \\
(7.48)\end{array}$ & $\begin{array}{c}4.26 \\
(4.20)\end{array}$ & $\begin{array}{c}19.9 \\
(19.1)\end{array}$ & 0.245 \\
\hline
\end{tabular}

cyanate groups of TDI, rapidly decreased and completely disappeared after $2.5 \mathrm{~h}$, as shown in Figure 2(c). The molecular weight of the prepolymer (II) was found to be 1830 (Calcd 1820). Preparation of the prepolymer (III) was confirmed by the disappearance of dimethylamino groups using amino group titration. The prepolymer (III) was allowed to react with $4,4^{\prime}$-bipyridine or 1,2-bis(4pyridyl)ethylene. With the proceeding of the reaction, the reaction solution turned from colorless to red, indicating that nitrogen atoms of the aromatic amines were quarternized by the prepolymer (III). Furthermore, the viscosity of the reaction solution increased. Elemental analyses and intrinsic viscosities $[\eta]$ of the elastomeric ionenes are shown in Table I. The experimental results from the elemental analyses are consistent with the calculated values. These results verify that the elastomeric ionenes can be prepared by the procedure shown in Figure 1.

The polymers obtained were red, transparent and elastic rubbery materials. The chemical structure of the elastomeric ionenes is characterized by alternating rigid polycation and flexible poly(oxypropylene) segments, and is analogous to segmented polyurethanes containing polycation segments as a hard segment.

\section{Conductivity of TCNQ Salts}

Figure 3 shows current-voltage characteristics of the TCNQ salts. In all samples, the response of current to the applied voltage was rapid and absorption current was hardly observed. This indicates that continuous conduction paths are formed in the TCNQ salts, despite the introduction of insulative poly(oxypropylene) chain in the elastomer-

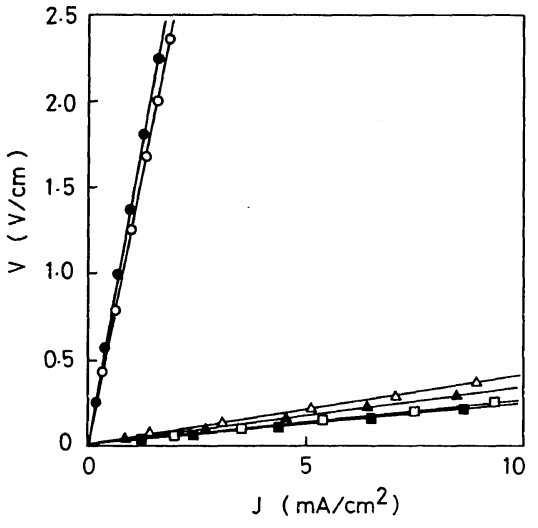

Figure 3. Current-voltage characteristics of TCNQ salts at $25^{\circ} \mathrm{C}$ : O, (BP-PPG)-TCNQ simple salt; $\triangle$, (BP-PPG)-TCNQ complex salt 0.5; $\square$, (BP-PPG)TCNQ complex salt 1.0; 9 , (BPE-PPG)-TCNQ simple salt; $\boldsymbol{A}$, (BPE-PPG)-TCNQ complex salt 0.75 ; (BPE-PPG)-TCNQ complex salt 1.0.

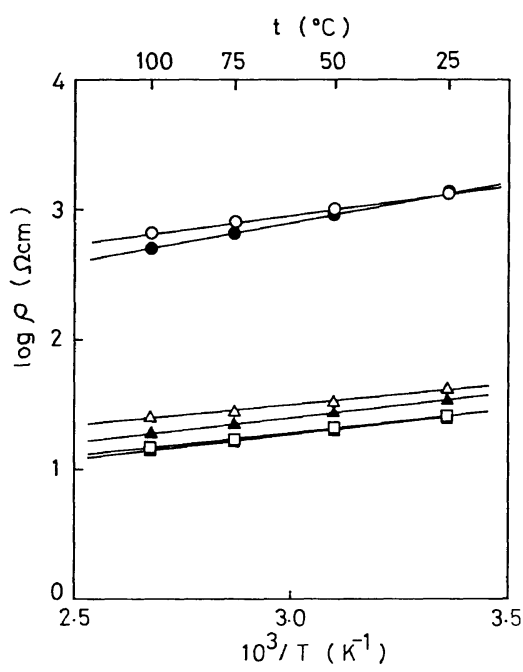

Figure 4. Relation between $\log \rho$ and inverse of absolute temperature for TCNQ salts: $O$, (BP-PPG)TCNQ simple salt; $\triangle$, (BP-PPG)-TCNQ complex salt

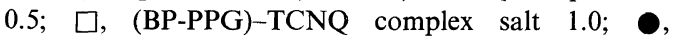
(BPE-PPG)-TCNQ simple salt; $\boldsymbol{\Delta}$, (BPE-PPG)TCNQ complex salt 0.75 ; $\square$, (BPE-PPG)-TCNQ complex salt 1.0 .

ic ionenes. In the simple salts, ohmic behavior was observed up to a current density at least of $2 \mathrm{~mA} \mathrm{~cm}^{-2}$, whereas in the complex salts, up to at least $10 \mathrm{~mA} \mathrm{~cm}^{-2}$. The values of resistivity $(\rho)$ were calculated from the slopes of the currentvoltage characteristics. 
Table II. Electronic properties of elastomeric ionene-TCNQ salts

\begin{tabular}{|c|c|c|c|}
\hline \multirow{2}{*}{ Sample } & \multirow{2}{*}{$\begin{array}{l}{\left[\mathrm{TCNQ}^{\circ}\right] /} \\
{\left[\mathrm{TCNQ}^{\circ}\right]}\end{array}$} & $\rho_{25^{\circ} \mathrm{C}}$ & \multirow{2}{*}{$\frac{E_{\mathrm{a}}}{\mathrm{eV}}$} \\
\hline & & $\Omega \mathrm{cm}$ & \\
\hline \multirow{3}{*}{ (BP-PPG)-TCNQ } & 0 & $1.28 \times 10^{3}$ & 0.091 \\
\hline & 0.5 & $4.12 \times 10^{1}$ & 0.070 \\
\hline & 1.0 & $2.67 \times 10^{1}$ & 0.077 \\
\hline \multirow{3}{*}{ (BPE-PPG)-TCNQ } & 0 & $1.41 \times 10^{3}$ & 0.123 \\
\hline & 0.75 & $3.46 \times 10^{1}$ & 0.103 \\
\hline & 1.0 & $2.48 \times 10^{1}$ & 0.079 \\
\hline
\end{tabular}

The relation between $\log \rho$ and inverse absolute temperature is shown in Figure 4. Since this relation was linear in the temperature range from $25^{\circ} \mathrm{C}$ to $100^{\circ} \mathrm{C}$, the values of $E_{\mathrm{a}}$ were calculated from the slopes. The values of $\rho$ at $25^{\circ} \mathrm{C}$ and those of $E_{\mathrm{a}}$ for the TCNQ salts are summarized in Table II. The reproducibility of the electrical data was excellent. For the simple salt, the values $\rho$ and $E_{\text {a }}$ were $\sim 10^{3}$ $\Omega \mathrm{cm}$ and $0.09-0.12 \mathrm{eV}$, respectively. They are fairly small, compared with the values of $\rho$ and $E_{\mathrm{a}}$ for other ionene-TCNQ simple salts. ${ }^{3,10,11} \mathrm{BP}$ or BPE in the elastomeric ionenes has high electron affinity and can be easily reduced to form colored species. ${ }^{12}$ The small $\rho$ and $E_{\mathrm{a}}$ values of the simple salts may be explained by an electron-transfer reaction from $\mathrm{TCNQ}^{-}$to $\mathrm{BP}$ or BPE which leaves $\mathrm{TCNQ}^{\circ}$ in the resulting structure. Similar results have been reported on the TCNQ salts from 4,4' ${ }^{\prime}$-bipyridine. ${ }^{13,14}$

The values of $\rho$ decreased by doping $\mathrm{TCNQ}^{\circ}$ in the simple salts, and took the minimum value at $\left[\mathrm{TCNQ}^{\circ}\right] /\left[\mathrm{TCNQ}^{-}\right]=1.0$. Consequently, the values of $\rho$ for the complex salts were $\sim 25 \Omega \mathrm{cm}$, which were relatively low among the polycation-TCNQ salts ever obtained. The values of $E_{\mathrm{a}}$ were $0.07-$ $0.08 \mathrm{eV}$. These results indicate the following facts. In complex salts, the $\mathrm{TCNQ}^{\circ}$ molecules are incorporated into the polymer matrix and the conductive moiety composed of $\mathrm{TCNQ}^{-}$and $\mathrm{TCNQ}^{\circ}$ is formed. Since the polarizable polycation segments in the elastomeric ionene are rigid and have the optimum intervals between $\mathrm{N}^{+}$atoms for the packing of the TCNQ molecules, an effective arrangement of the TCNQ molecules on conduction is formed under the influence of the structure of polycation segments. Since the polycation segment is separated by the poly(oxypropylene) segment, the

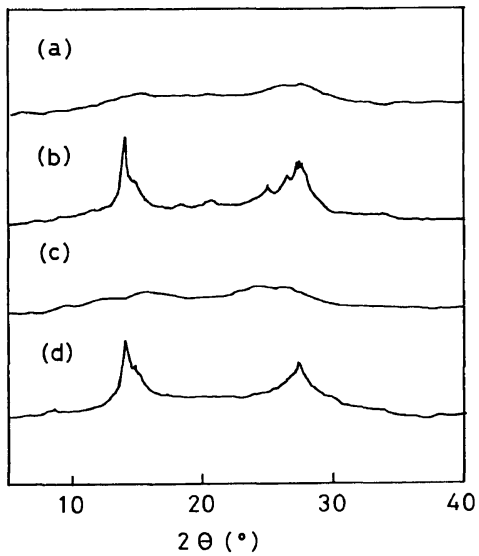

Figure 5. X-ray diffraction patterns of TCNQ salts: (a), (BP-PPG)-TCNQ simple salt; (b), (BP-PPG)TCNQ complex salt 1.0; (c), (BPE-PPG)-TCNQ simple salt; (d), (BPE-PPG)-TCNQ complex salt 1.0.

TCNQ moiety of a polycation segment is connected to the TCNQ moiety of a polycation segment of inter and/or intra polymer chain by strong interaction between $\mathrm{TCNQ}^{-}$and $\mathrm{TCNQ}^{\circ}$. As a result, a continuous conduction path is formed.

Figure 5 shows the X-ray diffraction patterns of the TCNQ salts. For the simple salts, only haloes characterizing amorphous materials were observed. On the contrary, for the complex salts, diffraction peaks were observed at $2 \theta=14^{\circ}$ (Bragg spacing of $6.33 \AA$ ) and $2 \theta=27^{\circ}$ (Bragg spacing of $3.30 \AA$ ).

In TCNQ salts of monomeric cations, segregated columns of TCNQ molecules and cations are generally formed. ${ }^{15}$ Ashwell et al. ${ }^{16}$ investigated the crystal structures of the complex salts of many biscations containing aromatic amines, and found that TCNQ molecules are stacked along the biscations and form a column structure. Murakami et $a l .{ }^{17}$ pointed out that in the X-ray diffraction patterns of TCNQ salts the diffraction peaks around $14^{\circ}$ were attributable mainly to planes parallel to the axes of the TCNQ columns and those around $27^{\circ}$ mainly to planes intersecting the column axes and nearly parallel to the molecular plane of TCNQ.

Applying these arguments the complex salts of the elastomeric ionenes, we can ascribe the diffraction peaks of the complex salts to the regularity in the arrangement of the TCNQ molecules. The TCNQ moiety composed of $\mathrm{TCNQ}^{-}$and $\mathrm{TCNQ}^{\circ}$ 
forms a stacking structure about the polycation segments. Continuous conduction columns, which are of course less regular than crystalline TCNQ salts of low molecular weight cations, are made by a sequence of these stackings. A microheterogeneous structure of these continuous conduction columns and the poly(oxypropylene) chain makes the complex salts of the elastomeric ionenes high conductive.

The TCNQ salts are soluble in organic solvents such as DMF and processible from solution as flexible films. The simple salts are dark purple and very flexible. The complex salts are black. Though the complex salts are stiffer than the simple salts, they are still flexible. The increase in stiffness can be attributed to the crystallinity of the complex salts. A microheterogeneous structure of the rigid TCNQ moiety and the flexible poly(oxypropylene) chain, which has a low glass transition temperature $\left(\sim-70^{\circ} \mathrm{C}\right)$, gives rise to these favorable mechanical properties.

\section{Change in Resistivity with Time}

Figure 6 shows the change in $\rho$ with time for the TCNQ salts in an ambient condition. The change in $\rho$ with time does not depend on the structure of the elastomeric ionenes and the simple and the complex salts, but $\rho$ increases slightly with time. The values of $\rho$ after a storage time of about 300 days were nearly twice as large as the initial values. For example, the values of $\rho$ for the (BPE-PPG)-TCNQ

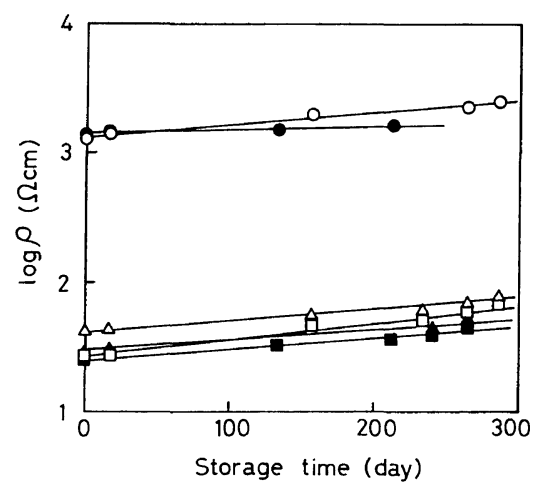

Figure 6. Change in resistivity $(\rho)$ with time for TCNQ salts in an ambient condition: $\square$, (BP-PPG)-TCNQ simple salt; $\triangle$, (BP-PPG)-TCNQ complex salt $0.5 ; \square$, (BP-PPG)-TCNQ complex salt 1.0; O. (BPE-PPG)TCNQ simple salt; $\boldsymbol{\Delta}$, (BPE-PPG)-TCNQ complex salt $0.75 ; \square$, (BPE-PPG)-TCNQ complex salt 1.0. complex salt $\left(\left[\mathrm{TCNQ}^{-}\right] /\left[\mathrm{TCNQ}^{\circ}\right]=1.0\right)$ went from $24.8 \Omega \mathrm{cm}$ to $45.0 \Omega \mathrm{cm}$ during this period of time, indicating the stability of these TCNQ salts.

\section{CONCLUSION}

In this paper, we have put forth to a method for obtaining highly conductive and very processible polycation-TCNQ salts. The method is outlined with synthesis of the complex salts of the elastomeric ionenes characterized by alternating rigid polycation and flexible poly(oxypropylene) segments. The polycation segments contribute to generating the high conductivity of the TCNQ salts, making a polarizable structure with an optimum intervals between $\mathrm{N}^{+}$atoms. The complex salts were obtained as flexible films with a high conductivity of $\sim 25 \Omega \mathrm{cm}$ in $\rho$ and $\sim 0.07 \mathrm{eV}$ in $E_{\mathrm{a}}$. A microheterogeneous structure of continuous conduction columns composed of $\mathrm{TCNQ}^{-}$and $\mathrm{TCNQ}^{\circ}$ and the poly(oxypropylene) chain brought about high conductivity and favorable mechanical properties.

Acknowledgement. This research was supported in part by a Grant-in-Aid for Scientific Research of the Ministry of Education, Science and Culture of Japan, for which the authors are grateful.

\section{REFERENCES}

1. J. H. Lupinski, K. D. Kopple, and J. H. Hertz, J. Polym. Sci., C, 16, 1561 (1967).

2. K. Mizoguchi, S. Kajiura, A. Nakano, E. Tsuchida, and I. Shinohara, Nippon Kagaku Kaishi, 1404 (1975).

3. T. Kamiya and I. Shinohara, J. Polym. Sci., Polym. Lett. Ed., 17, 641 (1979).

4. T. Kamiya, S. Tsuji, K. Ogatsu, and I. Shinohara, Polym. J., 11, 219 (1979).

5. T. Kamiya, K. Goto, and I. Shinohara, J. Polym. Sci., Polym. Chem. Ed., 17, 561 (1979).

6. T. Kamiya, M. Watanabe, K. Goto, N. Matsubara, and I. Shinohara, Polym. Prepr. Jpn., 27, 944 (1978).

7. L. R. Melby, R. J. Harder, W. R. Hertler, W. Mahler, R. E. Benson, and W. E. Mochel, J. Am. Chem. Soc., 84, 3374 (1962).

8. K. Mizoguchi, S. Tsuji, E. Tsuchida, and I. Shinohara, J. Polym. Sci., Polym. Chem. Ed., 16, 3259 (1978).

9. C. S. P. Sung and N. S. Schneider, Macromolecules, 8, 68 (1975).

10. R. Somoano, S. P. S. Yen, and A. Rembaum, $J$. Polym. Sci., B, 8, 467 (1970). 


\section{Conductivity of Elastomeric Ionene-TCNQ Salts}

11. S. Ikeno, M. Yokoyama, and H. Mikawa, Polym. J., 10, 123 (1978).

12. L. Michaelis and E. S. Hill, J. Gen. Phys., 16, 859 (1933).

13. A. Rembaum, V. Hadek, and S. P. S. Yen, J. Am. Chem. Soc., 93, 2532 (1971).

14. G. J. Ashwell, D. D. Eley, and M. R. Wills, J. Chem.
Soc., Faraday Trans. 2, 71, 1227 (1975).

15. I. F. Shchegolev, Phys. Status Solidi, Part A, 12, 9 (1972).

16. G. J. Ashwell, Phys. Status Solidi, Part B, 86, 705 (1978).

17. M. Murakami and S. Yoshimura, J. Phys. Soc. Jpn., 32, 488 (1975). 